\title{
Análise Fatorial Exploratória do Questionário de Percepção de Doenças Versão Breve (Brief IPQ)
}

\author{
Graziela Sousa Nogueira ${ }^{1}$ \\ Eliane Maria Fleury Seidl \\ Bartholomeu Torres Tróccoli \\ Universidade de Brasília
}

\begin{abstract}
RESUMO - O objetivo deste estudo foi proceder à análise fatorial exploratória da versão brasileira do Questionário de Percepção de Doenças Versão Breve (Brief IPQ). Participaram 325 pessoas adultas portadoras de enfermidades crônicas. A análise pelo método dos eixos principais, com rotação varimax, extraiu dois fatores: o Fator 1 (quatro itens; alfa de Cronbach $=0,80$ ) denominado representação emocional e o Fator 2 (três itens; alfa de Cronbach = 0,52) nomeado representação cognitiva. Embora a solução fatorial encontrada no presente estudo tenha diferido, em parte, da proposta teórica do Modelo de Autorregulação de Leventhal, a versão brasileira do Brief IPQ pode ser considerada válida e confiável para avaliar a percepção de doenças no contexto de enfermidades crônicas.
\end{abstract}

Palavras-chave: doença, doenças crônicas, percepção de doenças, análise fatorial, Brief IPQ

\section{Exploratory Factor Analysis of the Illness Perception Questionnaire Brief Version (Brief IPQ)}

\begin{abstract}
The objective of this study was to conduct an exploratory factor analysis of the Brazilian version of the Brief Illness Perception Questionnaire (Brief IPQ). Participants were 325 adults with chronic illnesses. A principal axis factoring analysis with varimax rotation extracted two factors: Factor 1 (four items, alpha $=0.80$ ) named emotional representation and Factor 2 (three items, alpha $=0.52$ ) named cognitive representation. Although the factor solution found in the present study differed in part from the Leventhal's Self-Regulation Model, the Brazilian version of the Brief IPQ can be considered a valid and reliable measure to assess the perception of illness in the context of chronic diseases.
\end{abstract}

Keywords: illness, chronic illness, illness perception, factor analysis, Brief IPQ

Observa-se um aumento no número de pesquisadores e profissionais dos campos da saúde e das ciências sociais que estão adotando conceitos e princípios das teorias de autorregulação para explicar o comportamento humano e promover mudanças de conduta em diferentes contextos (Leventhal, Brissete, \& Leventhal, 2003). Um dos modelos que tem recebido destaque na área da saúde é o Modelo de Autorregulação do Comportamento de Leventhal, proposto em 1980 e que derivou de estudos sobre o impacto de mensagens de medo sobre o comportamento preventivo (Quiceno \& Vinaccia, 2010; Sousa, 2003).

Esse modelo é centrado nas crenças individuais sobre saúde/doença e nas respostas às ameaças de doença (Quiceno \& Vinaccia, 2010; Sousa, 2003). Parte da premissa que o paciente é um agente ativo na resolução de seus problemas, respondendo de forma dinâmica à enfermidade segundo sua interpretação e avaliação (Husain, Dearman, Chaudhry, Rizvi, \& Waheed, 2008).

Segundo essa concepção, as respostas às doenças seguem três etapas. A primeira se refere à representação cognitiva da ameaça à saúde ou interpretação, na qual a pessoa enferma, ao ser confrontada com uma doença em potencial por meio de pistas internas (ex. sintomas) ou externas (ex. informação), tenta atribuir um significado ao problema, com base nas

1 Endereço para correspondência: Quadra 205, Praça Jandaia, Lote 05, Residencial Ingrid, apartamento 704, Águas Claras Sul, Brasília, DF, Brasil. CEP: 71925-000.E-mail: grazysousa@yahoo.com.br suas cognições preexistentes sobre a enfermidade. Essas cognições organizam-se em cinco dimensões: identidade (rótulos abstratos, ideias sobre a doença e interpretação dos sintomas); causa (atribuições do paciente acerca das prováveis causas da sua doença); dimensão temporal (duração do problema de saúde); consequências (crenças sobre a gravidade da doença e provável impacto no funcionamento biopsicossocial) e cura/controle (crença na cura/controle da doença e eficácia do tratamento) (Broadbent, Petrie, Main, \& Weinman, 2006; Cameron \& Leventhal, 2003; Diefenbach \& Leventhal, 1996; Figueiras, Machado, \& Alves, 2002; Leventhal et al., 2003; Pacheco-Huergo et al., 2011; Petrie \& Weinman, 2006; Santos, Pais-Ribeiro, \& Lopes, 2003; Sousa, 2003). A segunda etapa refere-se ao desenvolvimento e implementação das estratégias de enfrentamento. Nessa fase, a pessoa identifica e seleciona estratégias que lhe permitam adquirir o equilíbrio físico e emocional ameaçado ou perdido. Para tanto, pode desenvolver um conjunto de estratégias de enfrentamento que habitualmente se reúnem em dois grupos: enfrentamento de aproximação ou enfrentamento de evitação. Na terceira fase, ocorre a ponderação ou avaliação das estratégias de enfrentamento empregadas, com fins de adaptação à doença ou às suas consequências (Sousa, 2003; Pacheco-Huergo et al., 2011).

O Modelo de Autorregulação do Comportamento de Leventhal (Leventhal et al., 2003; Nerenz \& Leventhal, 1983) embasou a elaboração de instrumentos que tem 
como proposta avaliar a percepção de doenças. Assim, na tentativa de compreender a natureza da relação entre doença e estratégias de enfrentamento, bem como de desenvolver intervenções que permitam facilitar o autogerenciamento de enfermidades, estudiosos desenvolveram os seguintes instrumentos com base no Modelo de Autorregulação de Leventhal: (a) Illness Perception Questionnaire (IPQ) (Weinman, Petrie, Moss-Morris, \& Horne, 1996); (b) Revised Illness Perception Questionnaire (IPQ-R) (Moss-Morris et al., 2002); e (c) Brief Illness Perception Questionnaire (Brief $I P Q)$ (Broadbent et al., 2006).

Os autores do IPQ objetivaram construir um instrumento de avaliação, com um embasamento teórico estruturado a partir do Modelo de Leventhal (Leventhal et al., 2003; Nerenz \& Leventhal, 1983), que fosse suficientemente flexível para o uso de itens adicionais direcionados a grupos de pacientes, contextos e tratamentos de saúde específicos. Além disso, era essencial que esse instrumento tivesse boas propriedades psicométricas. O IPQ contém cinco escalas que avaliam os componentes da representação cognitiva da doença descritos anteriormente (identidade, causa, duração, consequências e cura/controle) (Weinman et al., 1996).

Porém, o uso da versão inicial do IPQ não tem sido recomendado, já que se encontra disponível uma versão revisada: o IPQ-R. Este foi desenvolvido com o propósito de sanar algumas limitações do IPQ constatadas por pesquisadores que fizeram uso do instrumento. Entre elas, cita-se o fato de não avaliar a representação emocional da doença e ainda por possuir debilidades em relação às propriedades psicométricas (Moss-Morris et al., 2002).

Apesar de suas vantagens, o IPQ-R é um instrumento extenso, com mais de cinquenta itens, o que dificulta sua utilização em contextos diversos, em especial na prática em saúde. Assim, outra versão do questionário foi desenvolvida, Brief IPQ (Broadbent et al., 2006). Trata-se de uma versão reduzida, que avalia a representação cognitiva e emocional das doenças, composta de nove itens desenvolvidos a partir do IPQ-R.

Destaca-se que, no artigo de Broadbent et al. (2006), não foi informada a estrutura fatorial da versão inglesa do Brief IPQ e seus indicadores psicométricos. Apesar dessa limitação, os autores apresentaram resultados de outras análises: confiabilidade teste-reteste, validade discriminante e validade preditiva. Para análise da confiabilidade testereteste, foram investigados pacientes ambulatoriais com doença renal. Eles responderam ao Brief IPQ e foram divididos em dois grupos, sendo novamente avaliados após três ou seis semanas. Para análise dos dados, foi utilizado o coeficiente de correlação de Pearson, que indicou que os itens possuíam boa confiabilidade teste-reteste, variando de 0,48 a 0,70 após três semanas e de 0,42 a 0,75 após seis semanas. A validade discriminante foi sustentada pela habilidade do instrumento para distinguir entre diferentes grupos de doenças. Para tanto, foram comparados os escores médios de pessoas com diabetes, asma, resfriado, pacientes que sofreram infarto do miocárdio antes de sua alta hospitalar e pacientes com dor no peito à espera do teste ergométrico. Foi realizada uma ANOVA one-way com comparações post hoc (Scheffe), que mostrou diferenças significativas entre as médias de doenças crônicas e agudas, indicando que o Brief
IPQ é sensível a variações na amostra e, assim, pode ser utilizado na investigação de percepção de doenças em pessoas com diferentes agravos de saúde. Para investigar a validade preditiva, foram avaliados pacientes que tiveram infarto do miocárdio após três meses. Com base na análise multivariada de variância, os autores afirmaram que componentes de representação da doença (como a presença de preocupação) funcionaram como preditores de comportamentos como um retorno mais lento ao trabalho em pacientes que tiveram infarto do miocárdio, por exemplo. Assim, os autores, apesar da não apresentação da estrutura fatorial do questionário, concluíram que a versão original do Brief IPQ é uma medida válida e fidedigna para avaliar a percepção de doenças, além de ser aplicável a uma grande variedade de enfermidades (Broadbent et al., 2006).

O Brief IPQ apresentou confiabilidade teste-reteste moderada em uma revisão sistemática de literatura que buscou identificar estudos sobre suas propriedades psicométricas em amostras de pessoas com desordens musculoesqueléticas (Leysen et al., 2015). Já um estudo norueguês encontrou evidências da validade e confiabilidade do Brief IPQ em pacientes com dor lombar crônica e aguda (Løchting, Garratt, Storheim, Werner, \& Grotle, 2013), no qual a consistência interna foi considerada satisfatória $(\alpha=0,72)$. Em um estudo feito com pacientes crônicos angolanos, o valor do alpha de Cronbach do Brief IPQ foi um pouco mais elevado do que no estudo norueguês, chegando a 0,77 (Cunha, 2014).

Ressalta-se que problemas com a versão holandesa do instrumento têm sido apontados na literatura. Um estudo holandês investigou a validade preditiva do Brief IPQ, considerando-a aceitável (Raaij, Schröder, Maissan, Pool, \& Wittink, 2012). Contudo, tais resultados foram questionados por outros pesquisadores (Van Oort, Schröder, \& French, 2011), que realizaram uma investigação enfocando os problemas durante o preenchimento da versão holandesa do Brief IPQ, sendo identificadas muitas dificuldades que levaram os autores a questionarem a validade de conteúdo do instrumento. Broadbent, Kaptein e Petrie (2011), por sua vez, também fizeram críticas à versão holandesa do Brief IPQ elaborada por Raaij et al. (2012), apontando problemas na tradução e na adaptação semântica dessa versão do instrumento, o que justificou as dificuldades de preenchimento da escala apontadas por Van Oort et al. (2011). Diante de tais controvérsias, Ng (2012) reforçou a importância de investigações acerca da validade de versões do Brief IPQ, traduzidas e adaptadas culturalmente para diferentes idiomas.

Ressalta-se que tais dificuldades parecem não limitar a utilização e aplicação da versão original do Brief IPQ, pois estudos apontam pontos positivos do instrumento: rápida e prática aplicação, pois seu preenchimento não se torna uma tarefa penosa e cansativa, favorecendo especialmente pessoas muito doentes e idosas; medida fácil de compreender e completar; resultados podem ser prontamente interpretados pelos pesquisadores e clínicos que fazem uso da mesma (Broadbent et al., 2006; Raaij et al., 2012). Além disso, constata-se a existência de estudos com diferentes objetivos realizados com pacientes em condições de saúde diversas, o que remete ao potencial de aplicação do Brief IPQ (Broadbent, Ellis, Thomas, Gamble, \& Petrie, 2009; Dalbeth 
et al., 2009; Rees, Leong, Crowston, \& Lamoureux, 2010; Sluiter \& Frings-Dresen, 2008; Živković, Buljan, Blajić, \& Šitum, 2008).

No Brasil, são inexistentes instrumentos validados com os mesmos objetivos e potencial de utilização do Brief IPQ. Em decorrência, considera-se a relevância para a psicologia da saúde de estudos que disponibilizem instrumentos validados para a prática clínica e de pesquisa, favorecendo a ampliação de conhecimentos sobre representações cognitivas e emocionais de doenças, com base no Modelo de Autorregulação de Leventhal. Assim, o presente estudo teve como objetivo proceder à análise fatorial exploratória da versão brasileira do Brief IPQ.

\section{Método}

\section{Participantes}

Participaram do estudo 325 pacientes, divididos em cinco grupos compostos de 65 indivíduos cada e definidos segundo o diagnóstico: HIV/aids, diabetes, hipertensão arterial, psoríase e asma. A decisão de compor a amostra com participantes acometidos por enfermidades diversas deveu-se ao propósito de validar um questionário de caráter genérico, aplicável a diferentes condições de adoecimento e não apenas a pessoas com um agravo específico. Na seleção por conveniência dos participantes, não foi feita distinção de sexo, raça, situação conjugal, condição socioeconômica e tempo de doença. Como critério de inclusão, no entanto, os participantes deveriam ter mais de 18 anos e ter cursado, no mínimo, a $4^{\mathrm{a}}$ série do ensino fundamental, tendo em vista a autoadministração do Brief IPQ na coleta de dados. Foram excluídos do estudo os pacientes que não consentiram em participar, bem como indivíduos com retardo mental ou outra limitação cognitiva que impossibilitasse o adequado preenchimento do instrumento.

Assim, em relação às características sociodemográficas dos participantes, $59,9 \%(n=194)$ eram do sexo feminino; a média de idade foi de 49,5 anos ( $D P=13,9$; amplitude 18 $85)$ e $61,1 \%(n=198)$ tinham companheiro(a). Em relação à escolaridade, houve predomínio de pessoas que tinham ensino fundamental incompleto $(35,8 \% ; n=116)$ e $56,6 \%$ $(n=183)$ não estavam trabalhando no momento da pesquisa. Sobre a renda familiar, a maioria recebia até três salários mínimos $(67,7 \% ; n=219)$ e $88 \%(n=285)$ relataram que possuíam uma religião vinculada a uma doutrina específica.

Em relação aos dados médico-clínicos da amostra, sobre o tempo de doença, $67 \%(n=217)$ revelaram que tinham mais de seis anos de diagnóstico; $64,5 \%(n=209)$ negaram internações por causa da doença; $64,8 \%(n=210)$ afirmaram que tinham alguma comorbidade e $77,2 \%(n=250)$ referiram que seguiam rigorosamente as orientações médicas, o que foi considerado indicador de adesão autorrelatada. Em relação à autoavaliação do estado geral de saúde, a média foi de 7,2 $(M d=8 ; \mathrm{AT}=0-10)$, sendo que $65,5 \%$ assinalaram números iguais ou superiores a sete, indicando que a percepção de estado de saúde variou de boa a excelente. $\mathrm{O}$ detalhamento dos dados sociodemográficos e médico-clínicos para o conjunto da amostra pode ser visualizado na Tabela 1.

\section{Instrumentos}

- Questionário sociodemográfico, elaborado para o estudo, com sete questões fechadas sobre sexo, idade, situação conjugal, escolaridade, situação laboral, renda familiar e religião.

- Questionário médico-clínico, elaborado para o estudo, para coleta de informações mediante questões fechadas e semiabertas sobre tempo de diagnóstico, número de internações, data da última internação, presença de comorbidades, adesão ao tratamento e avaliação do estado geral de saúde. Apenas essa última questão foi avaliada por meio de uma escala de dez pontos, onde 0 era igual a péssimo e 10 igual a excelente.

Tabela 1 Dados Sociodemográficos e Médico-clínicos da Amostra $(N=324)$

\begin{tabular}{|c|c|c|}
\hline & Variáveis & Frequência \\
\hline Sexo & $\begin{array}{l}\text { Feminino } \\
\text { Masculino }\end{array}$ & $\begin{array}{l}194(59,9) \\
130(40,1)\end{array}$ \\
\hline Escolaridade & $\begin{array}{l}\text { Ensino fundamental incompleto } \\
\text { Ensino fundamental completo } \\
\text { Ensino médio incompleto } \\
\text { Ensino médio completo } \\
\text { Ensino superior incompleto } \\
\text { Ensino superior completo }\end{array}$ & $\begin{array}{l}116(35,8) \\
40(12,3) \\
24(7,5) \\
82(25,3) \\
27(8,3) \\
35(10,8)\end{array}$ \\
\hline Renda & $\begin{array}{l}\text { Até um salário mínimo } \\
\text { De um a três salários mínimos } \\
\text { De quatro a seis salários mínimos } \\
\text { Mais de seis salários mínimos }\end{array}$ & $\begin{array}{c}90(27,8) \\
129(39,9) \\
72(22,3) \\
32(10)\end{array}$ \\
\hline Religião & $\begin{array}{l}\text { Com doutrina específica } \\
\text { Sem doutrina específica } \\
\text { Sem crenças religiosas } \\
\text { Outros }\end{array}$ & $\begin{array}{l}285(88) \\
27(8,3) \\
10(3,1) \\
2(0,6)\end{array}$ \\
\hline $\begin{array}{l}\text { Tempo de } \\
\text { diagnóstico }\end{array}$ & $\begin{array}{l}\text { Até seis meses } \\
\text { De sete meses a um ano } \\
\text { De dois a cinco anos } \\
\text { De seis a dez anos } \\
\text { De onze a vinte anos } \\
\text { Mais de } 21 \text { anos }\end{array}$ & $\begin{array}{c}22(6,8) \\
13(4) \\
72(22,2) \\
85(26,2) \\
82(25,3) \\
50(15,5)\end{array}$ \\
\hline $\begin{array}{l}\text { Número de } \\
\text { internações }\end{array}$ & $\begin{array}{l}\text { Nenhuma } \\
\text { Uma } \\
\text { Duas } \\
\text { Três } \\
\text { Quatro } \\
\text { Cinco } \\
\text { Seis ou mais }\end{array}$ & $\begin{array}{c}209(64,5) \\
51(15,7) \\
18(5,5) \\
6(1,9) \\
7(2,2) \\
3(0,9) \\
30(9,3)\end{array}$ \\
\hline $\begin{array}{l}\text { Adesão ao } \\
\text { tratamento } \\
\text { autorrelatada }\end{array}$ & $\begin{array}{l}\text { Sim } \\
\text { Não } \\
\text { Sem indicação de tratamento }\end{array}$ & $\begin{array}{c}250(77,4) \\
64(19,8) \\
10(2,8)\end{array}$ \\
\hline
\end{tabular}

Nota. A amostra passou a ser constituída de 324 casos devido à exclusão de um outlier multivariado. 
- Brief IPQ, instrumento traduzido por Nogueira (2012) da versão original em inglês (Broadbent et al., 2006) para a língua portuguesa, mediante técnicas de tradução reversa e análise semântica. Tal como a versão original, possui nove itens desenvolvidos a partir do IPQ-R (Moss-Morris et al., 2002). Todos os nove itens, exceto a questão causal (item 9), são respondidos utilizando uma escala de 0-10. O Brief IPQ é composto de cinco itens para avaliação da representação cognitiva da doença: consequências (item 1), dimensão temporal (item 2), controle individual (item 3), controle do tratamento (item 4) e identidade (item 5). Dois itens avaliam a representação emocional: preocupação (item 6) e emoções (item 8). Um item avalia a compreensão da doença (item 7). Para computar os escores, nessa versão original do instrumento, inverte-se os valores dos itens 3,4 e 7 , e adicionam-se os valores dos itens $1,2,5,6$ e 8 . Os escores variam de 0 a 80 e quanto mais alto o escore, maior a percepção de ameaça da doença. Por fim, a avaliação da representação causal (item 9) é feita por uma pergunta onde os pacientes informam os três fatores causais mais importantes associados à etiologia de sua doença, segundo sua interpretação. As respostas relacionadas a esse último item podem ser agrupadas em categorias (por exemplo: estilo de vida, estresse e hereditariedade), sendo então calculada a frequência das respostas (Broadbent et al., 2006). Ressalta-se que esse último item não foi incluído na análise fatorial exploratória, visto a sua característica qualitativa.

\section{Procedimentos}

Coleta de dados com os participantes. Pacientes de um hospital da rede pública de saúde do Distrito Federal foram convidados, no período de março a outubro de 2011, a participar voluntariamente do estudo enquanto aguardavam consultas em salas de espera ambulatoriais. Após aceitação e assinatura do Termo de Consentimento Livre e Esclarecido pelo paciente, o Brief IPQ foi autoadministrado, enquanto os questionários sociodemográfico e médico-clínico foram aplicados mediante entrevista individual. O tempo médio de resposta aos instrumentos foi de 15 minutos. A pesquisadora (primeira autora) juntamente com alunos de graduação em Psicologia devidamente treinados realizaram a coleta de dados.

Procedimentos preliminares à análise fatorial exploratória. Inicialmente, foi conduzida a análise preliminar dos dados e verificada a viabilidade da análise fatorial exploratória. Identificou-se a presença de missings (dados ausentes) em quatro casos, número que correspondeu a $1,23 \%$ da amostra, sendo que esses quatro participantes deixaram um item em branco do questionário Brief IPQ, representando um percentual muito baixo de dados perdidos considerando o total de itens do instrumento. Assim, foi realizada a substituição desses dados faltosos pelo valor da média do respectivo item, tendo em vista a adequação desse tipo de procedimento diante da baixa porcentagem de missings (Tabachnick \& Fidel, 2001). Para solução dos dois outliers univariados identificados foram realizadas alterações nos valores dos escores desses outliers, aproximando-os dos valores dos casos não outliers que se encontravam nas extremidades da distribuição, mantendo-se, portanto, o perfil de distribuição da amostra, tal como recomendado por Tabachnick e Fidel (2001).

Com base no cálculo da distância Mahalanobis, foram encontrados três casos de outliers multivariados extremos, mas como a sua exclusão não provocou mudanças relevantes na análise dos pressupostos e nos resultados finais, foram mantidos dois desses casos no banco de dados. Ressalta-se que esses outliers compartilhavam apenas duas características comuns: tinham o diagnóstico de asma e a mesma escolaridade (ensino fundamental incompleto). A decisão de excluir um caso multivariado extremo do banco de dados se deveu ao fato de se tratar daquele com o maior valor na distância Malahanobis $(30,5$ para o valor limite de 26,1) e porque esse participante respondeu sempre nos valores extremos, ou seja, zero ou dez nos itens do questionário, sugerindo que pode ter havido dificuldade de compreensão no preenchimento. Assim, o banco de dados final analisado foi composto de 324 casos, após a exclusão desse outlier multivariado.

O pressuposto estatístico de normalidade foi verificado por meio dos histogramas das oito variáveis métricas que compõem o Brief IPQ, dos valores de skewness e kurtosis, bem como por meio dos testes de normalidade KolmogorovSmirnov e Shapiro-Wilk. O pressuposto de normalidade foi violado, mas as transformações (logaritmo, raiz quadrada e inverso) não apresentaram resultados adequados, optandose, assim, por manter o banco de dados original. Gráficos de dispersão entre pares de variáveis não revelaram problemas de ausência de linearidade.

Houve indicação de multicolinearidade, sendo que o índice de condição (condition index) foi maior que 0,30 e pelo menos duas proporções das variâncias (variance proportion) estavam acima de 0,50 . Todavia, os valores de VIF (menores que cinco) e da tolerância (valores maiores que 0,1$)$ não indicaram problemas de colinearidade. Nesses casos, a multicolinearidade tende a não afetar o modelo (Hair, Black, Babin, Anderson, \& Tatham, 2009). Além disso, algum nível de multicolinearidade é desejável, uma vez que a análise fatorial trabalha com a matriz de correlações. Dessa forma, a análise de pressupostos indicou que a análise fatorial tinha viabilidade.

\section{Análise dos Dados}

Foi realizada análise estatística descritiva no que tange aos dados sociodemográficos e médico-clínicos. Para validação do instrumento, foi conduzida uma análise fatorial pelo método de extração dos eixos principais, rotação varimax (a primeira análise com rotação oblíqua não revelou correlações significativas entre os fatores). Procedeu-se ainda à análise de consistência interna pelo alfa de Cronbach. Foi utilizado o programa SPSS (Statistical Package for the Social Sciences, versão 18.5). Como critério de retenção de 
fatores, foi utilizada a análise paralela através da simulação de Monte-Carlo.

\section{Resultados}

Para a condução da análise fatorial foi realizada uma inspeção inicial da matriz de correlações (Correlation Matrix) e verificadas as possibilidades de fatorabilidade. A matriz foi considerada fatorável, já que a medida KMO (KaiserMeyer-Olkin) foi moderada $(0,74)$ e o Teste de Esfericidade de Bartlett's (qui-quadrado aproximado: 497,55; nível de significância de 0,01 ) indicou que a matriz correlacional não era uma matriz identidade. Ressalta-se que não foram encontrados problemas em relação à comunalidade, isto é, não foram observados valores extremos (0-1).

Com o intuito de confirmar o número de fatores a serem extraídos, conduziu-se a análise paralela através da simulação de Monte-Carlo. A escolha da análise paralela pode ser justificada pelo fato de a mesma diminuir a probabilidade de retenção equivocada de itens, por considerar o erro amostral e minimizar a influência do tamanho da amostra e das cargas

Tabela 2. Resultado da Análise Paralela Utilizando-se a Simulação Tipo Monte-Carlo

\begin{tabular}{ccc}
\hline Número de Fatores & Autovalor real & Autovalor aleatório \\
\hline 1 & 2,555 & 1,234 \\
2 & 1,597 & 1,144 \\
3 & 1,012 & $1,083^{*}$ \\
4 & 0,803 & $1,019^{*}$ \\
5 & 0,621 & $0,972^{*}$ \\
6 & 0,515 & $0,892^{*}$ \\
7 & 0,496 & $0,856^{*}$ \\
8 & 0,400 & $0,800^{*}$ \\
\hline
\end{tabular}

Nota. * Autovalor aleatório que supera o autovalor real. fatoriais dos itens (Damásio, 2012). Ao serem comparados os valores dos dados reais com os dos dados aleatórios, foram retidos dois fatores nos quais os autovalores dos dados reais eram maiores que dos dados aleatórios (Tabela 2).

Em seguida prosseguiu-se com a análise fatorial exploratória. Em uma primeira análise com rotação oblíqua (oblimin), foram extraídos dois fatores não correlacionados $(r=0,16)$, levando à decisão de proceder com a rotação varimax. O Fator 1 foi denominado representação emocional, composto pelos itens consequências (item 1), identidade (item 5), preocupações (item 6) e emoções (item 8). O Fator 2, intitulado representação cognitiva, agregou o controle individual (item 3), o controle do tratamento (item 4) e a compreensão (item 7). O item referente à dimensão temporal (item 2) apresentou cargas fatoriais muito baixas nos dois fatores, o que levou à decisão de sua exclusão do instrumento. A análise dos dois fatores separadamente revelou os seguintes valores do alfa de Cronbach: Fator $1 \mathrm{com}$ alfa de 0,80 e Fator 2 com alfa de 0,52. O resumo dos resultados da análise fatorial exploratória está na Tabela 3.

\section{Discussão}

O processo de validação do Brief IPQ envolveu a utilização de uma análise fatorial exploratória com extração pelo método dos eixos principais. Os fatores extraídos corresponderam à representação emocional (Fator 1) e representação cognitiva (Fator 2). Com base na estrutura do instrumento, segundo a qual cada item corresponde a um componente de representação da doença (cognitiva ou emocional), esperava-se que estes se agrupassem de acordo com o modelo teórico de Leventhal, o que ocorreu parcialmente. No Fator 1 agruparam-se dois itens de representação emocional (preocupação e emoções) e dois itens de representação cognitiva (consequências e identidade). Acredita-se que esses dois itens, originalmente na representação cognitiva, após o processo de tradução e

Tabela 3. Solução Fatorial com as Cargas Fatoriais, Comunalidades, Percentual de Variância Explicada e Alfas de Cronbach do Brief $I P Q$

\begin{tabular}{|c|c|c|c|c|}
\hline $\begin{array}{c}\text { No } \\
\text { item }\end{array}$ & Itens & F1 & F2 & h2 \\
\hline 1 & Quanto a doença afeta a sua vida? & 0,78 & & 0,70 \\
\hline 8 & $\begin{array}{l}\text { Quanto a sua doença o(a) afeta emocionalmente? (Por exemplo, faz você sentir raiva, } \\
\text { medo, ficar chateado ou depressivo). }\end{array}$ & 0,71 & & 0,50 \\
\hline 6 & Quanto você está preocupado(a) com a sua doença? & 0,68 & & 0,50 \\
\hline 5 & Quanto você sente sintomas (sinais, reações ou manifestações) da sua doença? & 0,67 & & 0,45 \\
\hline 3 & Quanto controle você sente que tem sobre a sua doença? & & 0,73 & 0,56 \\
\hline 4 & Quanto você pensa que o tratamento pode ajudar a melhorar a sua doença? & & 0,55 & 0,37 \\
\hline \multirow[t]{5}{*}{7} & Até que ponto você acha que compreende a sua doença? & & 0,40 & 0,16 \\
\hline & Autovalor & 2,55 & 1,60 & \\
\hline & Número de itens & 4 & 3 & \\
\hline & \% de variância explicada & & & \\
\hline & Alfa de Cronbach & & & \\
\hline
\end{tabular}

Nota. Fator 1: Representação emocional; Fator 2: Representação Cognitiva. 
adaptação cultural passaram a ter uma conotação mais voltada para a representação emocional em função dos verbos afetar ("Quanto a doença afeta a sua vida?") e sentir ("Quanto você sente sintomas da sua doença?"), o que justifica a migração de ambos para o fator representação emocional na versão brasileira, resultado que faz sentido do ponto de vista semântico e teórico. Os demais itens referentes à representação cognitiva (controle individual, controle do tratamento e compreensão) ficaram agrupados no Fator 2 - à exceção do item da dimensão temporal, que foi excluído -, resultado coerente se comparado à proposta de Leventhal. Não resta dúvida de que compreender a doença, perceber a capacidade de controle sobre a enfermidade e confiar no tratamento fazem sentido como aspectos de representação cognitiva.

Não era esperado que a dimensão temporal fosse excluída, o que ocorreu devido ao seu não agrupamento em nenhum dos dois fatores. Contudo, estudos prévios com outras versões do IPQ revelaram que esse item tem se mostrado problemático (Horne conforme citado por Moss-Morris et al., 2002). Investigações conduzidas com o IPQ original evidenciaram cargas fatoriais inferiores ao desejável em relação à dimensão temporal, sendo ressaltada a necessidade do aumento no número de itens para avaliá-la (Moss-Morris et al., 2002). Em estudos que avaliaram a confiabilidade teste-reteste do Brief IPQ, a dimensão temporal foi o item que apresentou o índice de correlação mais baixo $(0,35)$ em comparação com os outros itens da escala $(0,50$ a 0,87$)$, sendo assim o menos confiável (Leysen et al., 2015). Assim, são necessárias pesquisas que avaliem eventuais reestruturações na dimensão da temporalidade, já que esta não tem se mostrado satisfatória do ponto de vista empírico.

Outra limitação do item que avalia a dimensão temporal - o que provavelmente explique a sua fragilidade - referese à necessidade de alterar sua pontuação de acordo com o contexto no qual será utilizado. Em casos de doenças ou condições agudas, por exemplo, é esperado que pessoas com esse tipo de situação ou enfermidade percebam a sua doença como sendo de curta duração e assinalem os números próximos a zero em uma escala de 0-10, em que zero representa "muito pouco tempo". Já para pessoas com doenças crônicas, é desejável que assinalem os números mais próximos de dez, considerando que são doenças que tendem a durar "para sempre". Assim, uma pessoa que tem uma doença crônica e assinala dez na escala está sendo realista e não apresenta distorções cognitivas em relação a esse componente, mesmo que esse valor contribua para aumentar seu escore total. Já uma pessoa que marca números próximos de zero, acreditando que sua condição crônica será de curta duração, poderá ter uma percepção de doença menos centrada na ameaça, mas, contraditoriamente, parece apresentar crenças disfuncionais sobre a temporalidade.

Em função das limitações referentes a esse item, reiteradas pelas evidências empíricas, foi realizada sua reformulação de forma que não houvesse a perda de informação no uso do instrumento. Assim, na versão brasileira validada, disponibilizada em anexo, esse item (número 2 na escala original) passou a ser o item 8, avaliado em uma questão aberta, na qual as respostas serão analisadas qualitativamente e poderão ser agrupadas em categorias, como na questão nove. Instruções para análise dessa versão do instrumento também são encontradas em anexo.

Os dois fatores extraídos da análise dos eixos principais revelaram-se válidos, em decorrência das boas cargas fatoriais de seus itens. Foram encontradas cargas fatoriais variando de 0,40 a 0,78 , sendo que valores entre $\pm 0,30$ e \pm 0,40 são aceitáveis em estudos exploratórios e valores acima de $\pm 0,50$ são considerados necessários para a significância prática (Hair et al., 2009).

A consistência interna dos fatores 1 e $2(\alpha=0,80$ e $\alpha$ $=0,52$, respectivamente) foi considerada aceitável. Kline (2010) defende que o valor do alfa mais comumente aceito para testes cognitivos (como testes de inteligência) é 0,80; para testes de habilidade, o mais adequado é um alfa de 0,70 . Porém, esse estudioso afirma que, quando se trata de construtos psicológicos, valores abaixo de 0,70 podem ser esperados, por causa da diversidade dos conceitos que estão sendo medidos. Ademais, o número reduzido de itens do instrumento na versão breve, em especial do Fator 2, cujo alfa foi igual a 0,52 , pode constituir uma justificativa plausível para o valor mais baixo desse indicador (Damásio, 2012). Cabe ressaltar que o valor do alfa do Fator 2 não limita a confiabilidade do Brief IPQ, tendo em vista que as cargas fatoriais foram elevadas (apenas um item teve carga fatorial menor que 0,50$)$.

No artigo de validação do Brief IPQ em língua inglesa (Broadbent et al., 2006), não foi apresentada a estrutura fatorial do instrumento - o que dificulta a comparação com a presente análise -, sendo revelados apenas os resultados de confiabilidade teste-reteste, validade concorrente, validade preditiva e validade discriminante. Outros estudos que buscaram avaliar a validade e a confiabilidade do Brief IPQ também não apresentaram sua estrutura fatorial (Leysen et al., 2015; Løchting et al., 2013; Raaij et al., 2012; Van Oort et al., 2011). Assim, a ausência de informações sobre a estrutura fatorial do Brief IPQ foi um fator limitante para a discussão dos resultados encontrados na análise dos eixos principais do presente estudo.

\section{Considerações finais}

Diante das análises das propriedades psicométricas do Brief IPQ, conclui-se que se trata de um instrumento empiricamente válido para avaliar a percepção de doença de pessoas com enfermidades crônicas. Foram encontrados indicadores psicométricos confiáveis, de forma que o instrumento, em sua versão final, pode ser utilizado tanto em pesquisas empíricas quanto para orientar intervenções psicológicas no contexto da saúde, em especial as cognitivocomportamentais. Contudo, algumas limitações do estudo incluem a utilização de amostra não probabilística, trazendo limitações quanto à representatividade dos dados.

Sugere-se o desenvolvimento de estudos sobre o Brief IPQ em outras regiões do país e com outras populações (pessoas com outras enfermidades crônicas e com doenças agudas) para confirmar tais achados, bem como a condução de análises fatoriais confirmatórias. Ressalta-se que são escassos os estudos sobre essa temática no Brasil e esperase, com a disponibilização dessa ferramenta, contribuir 
para o aumento de pesquisas na área de saúde sobre o tema percepção de doenças à luz do Modelo de Autorregulação de Leventhal.

\section{Referências}

Broadbent, E., Ellis, C. J., Thomas, J., Gamble, G., \& Petrie, K. J. (2009). Can an illness perception intervention reduce illness anxiety in spouses of myocardial infarction patients? A randomized controlled trial. Journal of Psychosomatic Research, 67, 11-15. doi: 10.1016/j.jpsychores.2008.11.006

Broadbent, E., Kaptein, A. A., \& Petrie, K. J. (2011). Double Dutch: The 'think-aloud' Brief IPQ study uses a Dutch translation with confusing wording and the wrong instructions. British Journal of Health Psychology, 16(2), 246-249. doi: 10.1111/j.20448287.2011.02021.x.

Broadbent, E., Petrie, K. J., Main, J., \& Weinman, J. (2006). The Brief Illness Perception Questionnaire. Journal of Psychosomatic Research, 60, 631-637. doi:10.1016/j. jpsychores.2005.10.020

Cameron, L., \& Leventhal. H. (2003). Self-regulation, health, and illness. Em L. Cameron, \& H. Leventhal (Orgs.), The self regulation of health and illness behavior (pp. 1-13). Nova York: Routledge.

Cunha, M. C. A. (2014). O impacto das variáveis sociodemográficas e familiares nas crenças e na adesão ao tratamento: estudo exploratório numa amostra de doentes crônicos angolanos (Unpublished master's thesis). Retirado de http://hdl.handle. net $/ 10316 / 27904$

Dalbeth, N., Taylor, W. J., Chong, J., Leung, W, Chegudi, R., Horne, A.,.... Petrie, K. (2009). Illness perception in patients with gout. ACR/ARHP Annual Scientific Meeting, Philadelphia, USA. Retirado de http://acr.confex.com/acr/2009/ webprogram/ Paper10877.html

Damásio, B. F. (2012). Uso da análise fatorial exploratória em psicologia. Avaliação Psicológica, 11(2), 213-228.

Diefenbach, M. A., \& Leventhal, H. (1996). The common-sense model illness representation: Theoretical and practical considerations. Journal of Distress and Homeless, 5(1), 11-38.

Figueiras, M. J., Machado, V. A., \& C Alves, N.C. (2002). Os modelos de senso-comum das cefaléias crônicas nos casais: Relação com o ajustamento marital. Análise Psicológica, l(XX), 77-90.

Hair, J. F., Black, W. C., Babin, B. J., Anderson, R. E., \& Tatham, R. L. (2009). Análise multivariada de dados. Porto Alegre: Artmed.

Husain, M. O., Dearman, S. P., Chaudhry, I. B., Rizvi, N., \& Waheed, W. (2008). The relationship between anxiety, depression and illness perception in tuberculosis patients in Pakistan. Clinical Practice and Epidemiology in Mental Health, 4(1), 1-5. doi: 10.1186/1745-0179-4-4

Kline, R. B. (2010). Principles and practice of structural equation modeling. New York: The Guilford Press.

Leventhal, H., Brissette, I., \& Leventhal, E. A. (2003). The commonsense model of self-regulation of health and illness. In L. D. Cameron \& H. Leventhal (Eds.), The self-regulation of health and illness behavior (pp. 42-65). New York, NY: Routledge.
Leysen, M., Nijs, J., Meeus, M., van Wilgen, C. P., Struyf, F., Vermandel, A., ... Rousell, N. A. (2015). Clinimetric properties of Illness Perception Questionnaire Revised (IPQ-R) and Brief Illness Perception Questionnaire (Brief IPQ) in patients with musculoskeletal disorders: A systematic review. Manual Therapy, 20, 10-17. doi: 10.1016/j.math.2014.05.001

Løchting, I., Garratt, A. M., Storheim, K., Werner, E. L., \& Grotle, M. (2013). Evaluation of the Brief Illness Perception Questionnaire in sub-scute and chronic low back pain patients: Data quality, reliability and validity. Pain \& Relief, 2(122), 1-6. doi:10.4172/2167-0846.1000122

Moss-Morris, R., Weinman, J., Petrie, K. J., Horne, R., Cameron, L. D., \& Buick, D. (2002). The Revised Illness Perception Questionnaire (IPQ-R). Psychology and Health, 17(1), 1-16. doi:10.1080/08870440290001494

Nerenz, D. R., \& Leventhal, H. (1983). Self-regulation theory in chronic illness. In T. G Burish \& H. Leventhal (Eds.), Coping with chronic disease research and applications (pp. 13-47). New York, NY: Academic Press.

Ng, T. S. (2012). Brief Illness Perception Questionnaire (Brief IPQ). Journal of Physiotherapy, 58, 202-202.

Nogueira, G. S. (2012). Adaptação e validação do Brief Illness Perception Questionnaire (Brief IPQ) para a cultura brasileira (Unpublished marter's thesis). Retirado de http://repositorio. bce.unb.br/handle/10482/10777

Pacheco-Huergo, V., Vildrich, C., Pujol-Ribera, E., CabezasPeña, C., Núñez, M., Roura-Olmeda, P., ... Val, J. L. (2011). Percepción en enfermidades crônicas: Validación linguística del Illness Perception Questionnaire Revised y del Brief Illness Perception Questionnaire para la población española. Atención Primaria, 44, 280-287. doi:10.1016/j.aprim.2010.11.022

Petrie, K. J., \& Weinman, J. (2006). Why illness perceptions matter? Clinic Medical, 6, 536-539.

Quiceno, J. M., \& Vinaccia, S. (2010). Percepción de enfermedad: Uma aproximación a partir del Illness Perception Questionnaire. Psicología desde el Caribe, 25, 56-83.

Raaij, E. J., Schröder, C., Maissan, F. J., Pool, J. J., \& Wittink, H. (2012). Cross-cultural adaptation and measurement properties of the Brief Illness Perception Questionnaire- Dutch Language Version. Manual Therapy, 17, 330-335. doi: 10.1016/j. math.2012.03.001

Rees, G., Leong, O., Crowston, J. G., \& Lamoureux, E. L. (2010). Intentional and unintentional nonadherence to ocular hypotensive treatment in patients with glaucoma. Ophthalmology, 117(5), 903-908. doi: $10.1016 / \mathrm{j}$. ophtha.2009.10.038

Santos, C., Pais-Ribeiro, J., \& Lopes, C. (2003). Adaptação e validação do "Revised Illness Perception Questionnaire" (IPQ-R) em doentes oncológicos. Arquivos de Medicina, 17(4), 136-147.

Sluiter, J. K., \& Frings-Dresen, M. H. W. (2008). Quality of life and illness perception in working and listed chronic RSI patients. International Archives of Occupational and Environmental Health, 81, 495-501. doi: 10.1007/s00420-007-0222-z

Sousa, M. R. M. G. C. (2003). Estudo dos conhecimentos e representações de doença associados à adesão terapêutica nos diabéticos tipo 2 (Unpublished master's thesis). Retirado de http://repositorium.sdum.uminho.pt/handle/1822/668

Tabachnick, B. G., \& Fidel, L. S. (2001). Using multivariate statistics. San Francisco: Allyn \& Bacon. 
Van Oort, L., Schroder, C., \& French. D. P. (2011). What do people think about when they answer the Brief Illness Perception Questionnaire? A ‘think-aloud' study. The British Psychological Society, 16, 231-245. doi: 10.1348/135910710X500819

Weinman, J., Petrie, K. J., Moss-Morris, R., \& Horne, R. (1996). The Illness Perception Questionnaire: A new method for assessing the cognitive representation of illness. Psychology and Health, 11, 431-435. doi: 10.1080/08870449608400270
Živković, M. V., Buljan, M., Blajić, I., \& Šitum, M. (2008). Psychological status and illness perceptions in patients with melanoma. Collegium Antropological, 32(2), 75-78.

\section{Anexo \\ Questionário de Percepção de Doenças Versão Breve (Brief IPQ)}

Este questionário é sobre o que você pensa sobre a sua doença. Não existe resposta certa ou errada, o que importa é que você escolha a alternativa que melhor represente a maneira como você percebe a sua doença. Por exemplo:

Quanto você acha que é responsável pela sua saúde?

$$
\begin{array}{lllllllllll}
0 & 1 & 2 & 3 & 4 & 5 & 6 & 7 & 8 & 9 & 10
\end{array}
$$

Não sou

responsável

pela minha saúde

Sou totalmente responsável pela minha saúde

Para a questão acima, caso você pense que tem muita responsabilidade pela sua saúde, mas que não é totalmente responsável por ela, você poderia circular o número 8 ou o número 9 , por exemplo. Se você acha que tem pouca responsabilidade pela sua saúde poderia circular o número 1 ou o número 2 , e assim por diante

Da mesma maneira, para as questões a seguir, por favor, circule o número que melhor corresponda ao seu ponto de vista.

1. Quanto a doença afeta a sua vida?

$$
\begin{array}{lllllllllll}
0 & 1 & 2 & 3 & 4 & 5 & 6 & 7 & 8 & 9 & 10
\end{array}
$$

Não afeta em

nada a

minha vida

\section{Afeta}

gravemente a minha vida

2. Quanto controle você sente que tem sobre a sua doença

$$
\begin{array}{lllllllllll}
0 & 1 & 2 & 3 & 4 & 5 & 6 & 7 & 8 & 9 & 10
\end{array}
$$

Absolutamente

nenhum controle

Tenho extremo controle

3. Quanto você pensa que o tratamento pode ajudar a melhorar a sua doença?

$$
\begin{array}{lllllllllll}
0 & 1 & 2 & 3 & 4 & 5 & 6 & 7 & 8 & 9 & 10
\end{array}
$$

Não ajudará Ajudará

em nada

extremamente

4. Quanto você sente sintomas (sinais, reações ou manifestações) da sua doença?

$$
\begin{array}{lllllllllll}
0 & 1 & 2 & 3 & 4 & 5 & 6 & 7 & 8 & 9 & 10
\end{array}
$$

Não sinto

Sinto muito

nenhum sintoma sintomas graves

5. Quanto você está preocupado(a) com sua doença?

$$
\begin{array}{lllllllllllll} 
& 0 & 1 & 2 & 3 & 4 & 5 & 6 & 7 & 8 & 9 & 10 \\
\begin{array}{l}
\text { Nem um pouco } \\
\text { preocupado }
\end{array} & & & & & & & & & & & & \\
\hline
\end{array}
$$

6. Até que ponto você acha que compreende a sua doença?

$$
\begin{array}{lllllllllll}
0 & 1 & 2 & 3 & 4 & 5 & 6 & 7 & 8 & 9 & 10
\end{array}
$$

Recebido em 22.04.2013

Primeira decisão editorial em 23.08.2014

Versão final em 21.09.2014

Aceito em 05.05.2015

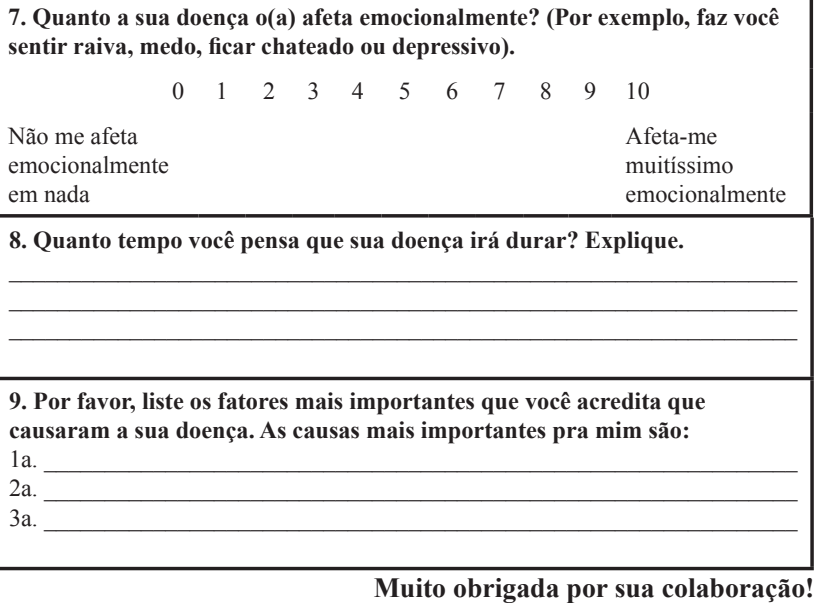

Instruções para Análise do Questionário de Percepção de Doenças Versão Breve (Brief IPQ)

Para computar os escores, primeiramente inverte-se as respostas dos itens 2, 3 e 6 (por exemplo, uma pessoa que marcou 1 terá o escore igual a 9 na questão, uma que marcou 2 será 8 , se marcou 3 será 7 , e assim por diante; o único valor que não é passível de inversão é 5). Os valores dos escores invertidos devem ser somados aos escores dos itens 1, 4, 5 e 7. Um escore alto reflete uma maior percepção de ameaça da doença (amplitude de 0-70). Quanto mais próximo de 70 for o escore, maior a percepção de ameaça imposta pela enfermidade. Sugere-se considerar que a pessoa tem uma percepção de relevante ameaça quando o escore for superior a 33 (a média dos sujeitos no estudo de validação foi utilizada como ponto de corte). Os itens abertos devem captar a resposta mais fidedigna possível, caso o preenchimento tenha sido mediante entrevista. No caso de pesquisas cientificas, quando se tem muitos casos, as respostas aos itens podem ser agrupadas em categorias, por exemplo, na questão causal: hereditariedade, sedentarismo, falta de informação etc. Ressalta-se que no que tange à questão causal é frequente que pessoas não consigam identificar três possíveis causas para a enfermidade, especialmente em casos de baixa escolaridade. Aconselha-se que seja registrado a ocorrência de ausência de resposta, pois tal dado indica a necessidade de intervenção psicoeducativa, já que sugere informação precária acerca da etiologia da doença. 